\title{
Lysozyme-Responsive Spray-Dried Chitosan Particles for Early Detection of Wound Infection
}

\author{
Claudia Tallian, ${ }^{\dagger}$ Gregor Tegl, ${ }^{*}{ }^{\dagger} \odot$ Lisa Quadlbauer, $^{\dagger}$ Robert Vielnascher, ${ }^{\dagger \dagger}$ Simone Weinberger, ${ }^{\ddagger}$ \\ Raymon Cremers, ${ }^{\S}$ Alessandro Pellis, $"$ Johannes W.O. Salari, ${ }^{\S}$ and Georg M. Guebitz ${ }^{\dagger, \ddagger}$
}

${ }^{\dagger}$ Institute for Environmental Biotechnology, Department for Agrobiotechnology (IFA-Tulln), University of Natural Resources and Life Sciences, Vienna (BOKU), Konrad-Lorenz-Strasse 20, 3430 Tulln an der Donau, Austria

${ }^{\ddagger}$ Austrian Centre of Industrial Biotechnology, Konrad-Lorenz-Strasse 20, 3430 Tulln an der Donau, Austria

${ }^{\S}$ Netherlands Institute for Applied Scientific Research, Eindhoven 5612 AP, The Netherlands

"Department of Chemistry, University of York, Heslington, York YO10 5DD, United Kingdom

\section{Supporting Information}

\begin{abstract}
Infections are a severe health issue, and the need for an early point-of-care diagnostic approach for wound infections is continuously growing. Lysozyme has shown a great potential as a biomarker for rapid detection of wound infection. In this study, spray-drying of labeled and derivatized chitosans was investigated for the production of small particles responsive to lysozyme. Therefore, various chitosans, differing in their origin (snow crab, Chionoecetes sp., with medium and low molecular weight or shrimp) were $N$-acetylated, labeled with reactive black 5 , and tested for solubility and spray-drying suitability. Reactive black-5-stained $\mathrm{N}$-acetylated chitosan (low molecular weight, origin crab) was successfully spray-dried, and the obtained particles were characterized regarding size, $\zeta$ potential, and morphology. The particles showed an average hydrodynamic radius of $612.5 \pm 132.8 \mathrm{~nm}$. $\zeta$ potential was measured in the context of a later application as an infection detection system for wound infections in artificial wound fluid $(-6.14 \pm 0.16 \mathrm{mV})$ and infected wound fluid $(-7.93 \pm 1.35 \mathrm{mV})$. Furthermore, the aggregation behavior and surface structure were analyzed by using scanning electron microscopy and confocal laser scanning microscopy revealing spherical-shaped particles with explicit surface topologies. Spray-dried $N$-acetylated chitosan particles showed a 5-fold increase in lysozyme-responsive release of dyed chitosan fragments due to the enhanced surface area to volume ratio when compared to non-spray-dried $\mathrm{N}$-acetylated chitosan flakes. On the basis of these results, the study showed the improved properties of $\mathrm{N}$-acetylated spray-dried chitosan particles for future applications for early and rapid infection detection.
\end{abstract}

KEYWORDS: wound infection, $N$-acetylated chitosan, lysozyme, diagnostics, $N$-acetylation, enzyme-responsive release

\section{INTRODUCTION}

Worldwide, wound infections are a severe health issue with 7$10 \%$ of all surgical wounds yearly diagnosed as infected and over 6.5 million patients suffering from chronic wounds in the United States only in 2009. ${ }^{1,2}$ Chronic infections develop from traumatic or surgical wounds and health-care-associated infections with life-endangering consequences such as sepsis; ${ }^{3}$ thus, reliable infection prevention, detection, and treatment strategies are needed.

In general, wound healing is a complex process involving different phases of hemostasis, inflammation, proliferation, and remodeling. ${ }^{4}$ Microbial colonization of the wound is possible during all phases, and most infections are identified as polymicrobial. ${ }^{5}$ In the case of infection, the host defenses are not capable of competing against the invading organisms resulting in delayed wound healing, in increased patient trauma, and possibly in the development of chronic wounds. ${ }^{6,7}$
In this context, infections have to be identified rapidly and early enough, whereby signs for clinical diagnosis were described by Cutting and Harding. Delayed healing, offensive odor, discoloration, increase in lesion size, pain or discomfort, and prolonged exudate production were described as important signs for the diagnosis of infection. ${ }^{8}$ However, clinical signs are still seen as controversial, as discussed by Gardner et al. ${ }^{9,10}$ Therefore, microbiological sampling combined with modern molecular biology tools, hematologic analysis, and the analysis of biochemical markers such as bacteria, metabolites, or enzymes offer additional potential for reliable and rapid diagnosis. ${ }^{11}$

Received: January 9, 2019

Accepted: February 19, 2019

Published: February 19, 2019 
Various methods for the detection of enzymes as biochemical markers are described, and recently reviewed by Tegl et al. ${ }^{12}$ comprising aptamers or amperometric sensors, color reactions, antibody trapping, and dye release systems, among others. ${ }^{6,12}$ These systems are based on elevated activities of certain enzymes in human wound fluid of infected wounds, whereby these enzyme activities refer to the current wound status. Biomarkers such as lysozyme, human neutrophil elastase (HNE), and myeloperoxidase (MPO) are frequently discussed in this regard ${ }^{13}$ while among these enzymes only lysozyme is exclusively produced by the human immune system, as a direct reflection of the host's immune response. ${ }^{14}$ Considerably increased activity of lysozyme in postoperative wounds and decubitus wound fluids was reported by Hasmann et al. $(4830 \pm 1848 \mathrm{U} / \mathrm{mL}$; compared to noninfected wounds, $376 \pm 240 \mathrm{U} / \mathrm{mL})$, which demonstrated the potential of lysozyme as the basis for the development of new infection detection systems. $^{15,16}$

Lysozymes are glycoside hydrolases found in $\mathrm{GH}$ families $19,22,23,24$, and 46 , commonly hydrolyzing the glycosidic linkage between the $N$-acetyl glucosamine and $N$-acetylmuramic acid units of peptidoglycan of bacterial cell walls. ${ }^{17}$ Additionally, lysozyme was described to hydrolyze chitosan, a biopolymer consisting of $\beta(1,4)$-linked units of $N$-acetyl glucosamine and glucosamine, ${ }^{17}$ which offers an alternative lysozyme-responsive biomaterial overcoming the bacterial origin of peptidoglycan and therefore the possibility of an immune response., 12 The hydrolysis rate of chitosan by lysozyme is influenced by the physicochemical properties of the chitosans such as the degree of $N$-acetylation (DA) as well as the distribution of acetyl groups along the polymer chain (PA) ${ }^{18}$ Chemical modifications of chitosans successfully led to the development of chitosan-based lysozyme substrates, enabling the detection of increased lysozyme activities in human wound fluids. ${ }^{19}$ Furthermore, chitosan and chitosan derivates have been extensively studied regarding biocompatibility and biotoxicity. ${ }^{20,21}$ Published studies on chitosan toxicity showed a strong dependency on the type of chemical modification and application routes. ${ }^{21-23}$ Nonetheless, chitosan is widely regarded as being a nontoxic material, and products in dietary applications are approved in Japan, Italy, and Finland. Additionally, chitosan as wound dressing material was already approved by the FDA. ${ }^{21}$

On the basis of this previous report, the present work investigates the synthesis of a functionalized chitosan-based material suitable for spray-drying, resulting in particles that enable a simple and rapid visual lysozyme detection in wound fluids. Chitosans of different properties were chemically modified to ensure high sensitivity toward lysozyme degradation and, at the same time, have efficient processability for spray-drying. The chitosan starting materials were characterized, modified by $\mathrm{N}$-acetylation, and labeled using reactive black 5. The spray-dried enzyme substrate offered a secondgeneration substrate for the detection of lysozyme, greatly accelerating the visual response of in vitro infection detection.

\section{MATERIALS AND METHODS}

Materials. Crab chitosans 90/5 of Chionoecetes sp. (low molecular weight; LMW) was purchased from Heppe Medical Chitosan GmbH; chitosans from shrimp shells and from crab shells (80/20, medium molecular weight; MMW) were purchased from Sigma-Aldrich. All other chemicals were of analytical grade from Sigma-Aldrich and used as received, if not otherwise stated.
Size Exclusion Chromatography (SEC) Characterization of Chitosans. Purchased chitosans (crab LMW, shrimp and crab MMW) were characterized regarding the number-average molecular weight $\left(M_{\mathrm{n}}\right)$, the weight-average molecular weight $\left(M_{\mathrm{w}}\right)$, and the polydispersity $(\mathrm{PD})$ via size exclusion chromatography. Samples were dissolved in a mixture of acetate buffer with $0.15 \mathrm{M}$ acetic acid, $0.1 \mathrm{M}$ sodium acetate, and $0.4 \mathrm{mM}$ sodium azide in ultrapure water (mobile phase) to a final concentration of $1.0 \mathrm{mg} / \mathrm{mL}$. An Agilent 1100 Series chromatography system (Agilent Technologies) equipped with an Agilent 1200 G1362A refractive index detector (Agilent Technologies) and a TSKgel G5000PWxl column (Tosoh Bioscience, Montgomeryville, PA) was used for the analysis. System calibration was performed using a pullulan standard (Fluka) in the $342 \mathrm{Da}$ to 800 $\mathrm{kDa}$ range. The injection volume was $100 \mu \mathrm{L}$ and the mobile phase flow rate $0.3 \mathrm{~mL} / \mathrm{min}$ at $21{ }^{\circ} \mathrm{C}$.

Synthesis of $\mathbf{N}$-Acetylated Chitosans. $\mathrm{N}$-Acetylation of chitosans (crab LMW, shrimp and crab MMW) was performed according the procedure of Tegl et al. ${ }^{19}$ Briefly, the chitosans were dissolved in $10 \%$ acetic acid to a final concentration of $1 \% \mathrm{w} / \mathrm{v}$ followed by a 1:1 dilution in ethanol. The mixtures were then stirred for $10 \mathrm{~min}$ at $21^{\circ} \mathrm{C}$. Subsequently, acetic anhydride was added in the concentrations of $0.50,0.75,1.00$, and $1.25 \mathrm{~mol}$ equivalent. The calculation was based on the glucosamine units of the polymers. The mixtures were further stirred for $1 \mathrm{~h}$, and the $\mathrm{pH}$ was adjusted to 8.0 using $1 \mathrm{M} \mathrm{NaOH}$. $\mathrm{N}$-Acetylated chitosans were then washed to a neutral $\mathrm{pH}$ with $\mathrm{ddH}_{2} \mathrm{O}$ and lyophilized prior to use.

Nuclear Magnetic Resonance Spectroscopy (NMR). The degree of acetylation (DA) was determined by using ${ }^{1} \mathrm{H}$ nuclear magnetic resonance on a Bruker Avance II 400 spectrometer, with a resonance frequency for ${ }^{1} \mathrm{H}$ of $400.13 \mathrm{MHz}$. The system was equipped with a $5 \mathrm{~mm}$ observe broadband probe head with $z$-gradients. Samples were dissolved in a mixture of $\mathrm{D}_{2} \mathrm{O}$ and $1 \%$ acetic acid- $\mathrm{D}_{4}$, if not otherwise specified. DAs were calculated using the integrals of the proton peaks $\mathrm{H}_{2}$ of the deacetylated monomer and the three proton peaks of the acetyl groups, according to the protocol published by Tegl et al. ${ }^{19}$ (eq 1).

$$
\mathrm{DA}[\%]=\left(1-\left(\frac{\mathrm{H} 2 \mathrm{D}}{\mathrm{H} 2 \mathrm{D}+\frac{\mathrm{HAc}}{3}}\right)\right) \times 100
$$

Here, $\mathrm{H} 2 \mathrm{D}$ is the integral of the $\mathrm{H} 2$ proton, and HAc is the summation of integrals of the $\mathrm{N}$-acetyl group.

Attenuated Total Reflection Fourier Transform Infrared Spectroscopy (ATR-FTIR). N-Acetylated chitosan samples were analyzed via Fourier transform infrared spectroscopy in attenuated reflection mode (ATR-FTIR) using a Spectrum 100 PerkinElmer FTIR spectrometer (Waltham, MA). Spectra were normalized between 950 and $1050 \mathrm{~cm}^{-1}$, baseline-corrected, and compared with spectra of untreated chitosans. The amide I band at $1640 \mathrm{~cm}^{-1}$ and the amide II band at $1560 \mathrm{~cm}^{-1}$ were considered for the analysis of the DA. ${ }^{19}$

Preparation of Chitosan Substrates. Chitosan substrates for further spray-drying were produced by covalent staining of $\mathrm{N}$ acetylated chitosans with reactive black 5 (RB5). Therefore, $100 \mathrm{mg}$ of $\mathrm{N}$-acetylated chitosans was swollen in ultrapure water for $20 \mathrm{~min}$. Afterwards, a $0.5 \%(\mathrm{w} / \mathrm{v})$ solution of RB5 in ultrapure water and a solution of $2.5 \%(\mathrm{w} / \mathrm{v}) \mathrm{Na}_{2} \mathrm{SO}_{4}$ and $1 \%(\mathrm{w} / \mathrm{v}) \mathrm{NaCO}_{3}$ were added to the swollen chitosans. The solution was incubated for $10 \mathrm{~min}$ at $21^{\circ} \mathrm{C}$, followed by $1 \mathrm{~h}$ at $65{ }^{\circ} \mathrm{C}$. After incubation, the samples were centrifuged and rinsed with ultrapure water to remove unbound stain. Samples were lyophilized and stored until further use.

Spray-Drying of Chitosan Substrates. Spray-drying was performed with a Büchi Mini spray-dryer B-290. First, aqueous solutions of the modified chitosan substrates were prepared. The solvent was 1 wt $\%$ acetic acid solution in water. The 0.5 wt \% chitosan substrate solutions were prepared in the acidic solvent. The chitosan solution was stirred until a clear solution was obtained. The solutions were filtered through a $5 \mu \mathrm{m}$ filter before spray-drying. The chitosan solution was then sprayed in the spray-dryer with pump 
Table 1. Experimental Overview of Tested Chitosans ${ }^{a}$

\begin{tabular}{|c|c|c|c|c|c|c|c|c|c|c|}
\hline chitosan origin & $M_{\mathrm{n}}[\mathrm{kDa}]^{b}$ & $M_{\mathrm{w}}[\mathrm{kDa}]^{b}$ & $\mathrm{PD}^{b}$ & RB5 staining & solubility & $\mathrm{SD}$ & lysozyme release & $R_{\mathrm{H}}[\mathrm{nm}]$ & $\mathrm{DA}^{c}[\%]$ & ID \\
\hline \multirow[t]{4}{*}{ crab MMW } & $485.5 \pm 3.7$ & $1834.2 \pm 203.5$ & $3.78 \pm 0.39$ & + & - & - & - & - & 36 & A \\
\hline & & & & + & - & - & - & - & 37 & B \\
\hline & & & & + & - & - & - & - & 40 & $\mathrm{C}$ \\
\hline & & & & - & - & - & - & - & n.d. & $\mathbf{D}$ \\
\hline \multirow[t]{4}{*}{ shrimp } & $236.6 \pm 4.5$ & $835.4 \pm 27.6$ & $3.53 \pm 0.05$ & + & - & - & - & - & 36 & $\mathbf{E}$ \\
\hline & & & & + & - & - & - & - & 46 & $\mathbf{F}$ \\
\hline & & & & - & - & - & - & - & n.d. & G \\
\hline & & & & - & - & - & - & - & n.d. & $\mathbf{H}$ \\
\hline \multirow[t]{4}{*}{ crab LMW } & $121.8 \pm 7.0$ & $370.5 \pm 74.2$ & $3.07 \pm 0.81$ & + & - & - & - & - & 37 & I \\
\hline & & & & + & + & + & + & $612.5 \pm 132.8$ & 49 & $\mathbf{J}$ \\
\hline & & & & - & - & - & - & - & 51 & $\mathbf{K}$ \\
\hline & & & & - & - & - & - & - & n.d. & $\mathbf{L}$ \\
\hline
\end{tabular}

${ }^{a} M_{\mathrm{n}}$ refers to the number-average molecular weight in $\mathrm{kDa}, M_{\mathrm{w}}$ to weight-average molecular weight, $\mathrm{PD}$ to polydispersity, $\mathrm{DA}$ to the degree of acetylation in \%, RB5 staining to the staining with reactive black 5, SD to spray-drying, RH to the hydrodynamic radius in $\mathrm{nm}$, ID to the sample ID, and n.d. to not determined. \pm values indicate the success of the performed experiments for the tested material. ${ }^{b}$ Calculated via SEC using pullulan standards. ${ }^{c}$ Calculated via ${ }^{1} \mathrm{H}$ NMR.

setting $10(3.3 \mathrm{~mL} / \mathrm{min})$. The solution droplets were dried to form particles under the following conditions. A nitrogen flow of $\sim 0.1 \mathrm{~L} / \mathrm{s}$ passed through the drying column. The aspirator setting was $70 \%$ and the pressure 50 mbar. The inlet temperature of the gas flow was $205^{\circ} \mathrm{C}$. Chitosans powder particles were produced with a yield of $\sim 59 \%$.

Scanning Electron Microscopy (SEM). The surface morphology of $\mathrm{N}$-acetylated chitosan flakes and spray-dried $\mathrm{N}$-acetylated chitosan particles was determined using SEM. All images were acquired by collecting secondary electrons on a Hitachi 3030TM instrument (Metrohm INULA GmbH) working at energy-dispersive X-ray spectrometry (EDX) acceleration voltage. For the comparison of dynamic-light-scattering-based (DLS-based) size distribution with obtained SEM results, the pictures were further processed by the use of ImageJ software for particles smaller than $3 \mu \mathrm{m}$ to investigate the nanofraction of the samples. ${ }^{24}$ The obtained data on size were processed using ImageJ software ${ }^{24}$ to calculate the relative frequencies of the sub-micro-sized particles and the microfraction of stained $\mathrm{N}$ acetylated chitosan particles.

Laser Doppler Velocimetry (LDV). Laser Doppler velocimetry (LDV) was used to determine the electrophoretic mobility and calculate the $\zeta$ potential $(\zeta)$ of spray-dried chitosan particles using the Henry equation: ${ }^{25}$

$$
U_{\mathrm{E}}=\frac{2 \varepsilon \zeta f(\mathrm{Ka})}{3 \eta}
$$

Here, $\zeta$ is the $\zeta$ potential $(\mathrm{mV}), U_{\mathrm{E}}$ the electrophoretic mobility $\left(\mathrm{m}^{2} /(\mathrm{s} \mathrm{V})\right), H$ the viscosity $(\mathrm{Pa} \mathrm{s}), \varepsilon$ the dielectric constant $(\mathrm{F} / \mathrm{m})$, and $f(\mathrm{Ka})$ Henry's function.

For data evaluation, the Smoluchowski approximation ${ }^{26}$ was used, whereby $f(\mathrm{Ka})$ is defined as 1.5. Therefore, $\zeta$ of chitosan particles was measured using Malvern Zetasizer Nano ZS (Malvern Instruments $\mathrm{GmbH}$, Germany), with a $15 \times$ dilution in $1 \times$ PBS buffer, in artificial wound fluid (AWF), human serum albumin $2 \% \mathrm{w} / \mathrm{v}$, sodium chloride $111.5 \mathrm{mM}$, sodium hydrogen carbonate $17.5 \mathrm{mM}$, sodium lactate 11 $\mathrm{mM}$, glucose $1.2 \mathrm{mM}$, calcium chloride $2.2 \mathrm{mM}$, magnesium chloride $0.9 \mathrm{mM}$, urea $9 \mathrm{mM}$, and potassium chloride $4.4 \mathrm{mM}, \mathrm{pH} 7.2$ with an average lysozyme activity of $83.33 \mu \mathrm{kat} / \mathrm{mg}$ and in $1: 10$ diluted wound fluids (WF) in $0.9 \% \mathrm{NaCl}$ solution. For each sample three measurements with a minimum of 20 runs were averaged, and for each measurement the optimum attenuator position and number of runs were optimized automatically. Outliers were tested using a modified Thompson $\tau$ test and excluded from results.

Dynamic Light Scattering (DLS). Hydrodynamic radius $\left(R_{\mathrm{H}}\right)$ was measured using dynamic light scattering (DLS) performed with a Wyatt DynaPro NanoStar detector (Wyatt Technology Europe). Measurements were performed in disposable cuvettes with diluted spray-dried chitosan particle samples in $66 \mathrm{mM}$ potassium phosphate buffer $\mathrm{pH}$ 6.2. Samples were measured three times with 20 acquisitions and $2 \mathrm{~s}$ acquisition time for each measurement. Obtained results were statistically tested for outliers using a modified Thomson $\tau$ test and averaged.

Confocal Laser Scanning Microscopy (CLSM). Staining efficiency was determined by the visualization of the dye distribution using a FV1000 Fluoview (Olympus) confocal laser scanning microscope equipped with an UPLSAPO $60 \times \mathrm{W}$ aperture objective (Olympus). For fluorescent imaging of stained chitosan substrates and spray-dried particles a laser light wavelength of $405 \mathrm{~nm}\left(\lambda_{\text {em }}\right.$ 426-526 nm) was used. The signal/noise ratio of each individual channel, sample and field of view, the laser intensity, the photomultiplier gain, as well as the offset were optimized for each sample individually. Pictures of each sample were taken from the fluorescent (F) and the bright field (BF) microscopy channel, additionally to the corresponding overlay (OVL). The obtained data on particle size were evaluated using ImageJ software. ${ }^{24}$

Lysozyme Activity Assay. The determination of lysozyme activity was based on the method of Shugar et al. ${ }^{27}$ Therefore, cells of Micrococcus lysodeikticus were suspended in $66 \mathrm{mM}$ potassium phosphate buffer $\mathrm{pH} 6.2$ to obtain a final concentration of $0.01 \%(\mathrm{w} /$ v). For UV/vis spectroscopy analyses, $290 \mu \mathrm{L}$ of bacteria suspension was mixed with lysozyme solutions of different concentrations (0.1, $0.05,0.01,0.0075,0.005,0.0025,0.001 \mathrm{mg} / \mathrm{mL}$ ), and the increasing transparency was measured at $450 \mathrm{~nm}$ using a Tecan Infinite 200 Pro plate reader (Tecan Trading AG) for $10 \mathrm{~min}$ at $25{ }^{\circ} \mathrm{C}$. For calculations of the enzyme activity one unit of lysozyme was defined to decrease the absorbance at $450 \mathrm{~nm}$ by 0.001 per minute at $\mathrm{pH} 7.0$.

Wound Fluid (WF) Activity Assay. The activity of wound fluids ${ }^{19}$ was measured with the same method as lysozyme activity. Used WFs ( $\mathrm{A}$ and $\mathrm{B}$ ) were tested, whereby $\mathrm{WF}_{\mathrm{A}}$ clinically described as noninfected ${ }^{15,16}$ had low lysozyme activity and was chosen as negative control. The permission for wound fluid collection and scientific usage was obtained from Medisch Ethische ToetsingCommissie Twente (Netherlands, Enschede) under the statement number METC/14213.haa.

Enzyme-Responsive Degradation Studies of ChitosanBased Substrates. Produced chitosan substrates with covalently bound RB5 as well as spray-dried and stained chitosan particles were tested regarding lysozyme-responsive color release. Therefore, $2 \mathrm{mg}$ of each sample was suspended in $0.5 \mathrm{~mL}$ of $1 \mathrm{mg} / \mathrm{mL}$ lysozyme solution with an average activity of $83.333 \mu \mathrm{kat} / \mathrm{mg}$ in $66 \mathrm{mM}$ potassium phosphate buffer $\mathrm{pH} 6.2$, in AWF or in 1:10 diluted WF in $0.9 \% \mathrm{NaCl}$ solution. Experiments were performed in triplicates. After suspension, samples were incubated for $24 \mathrm{~h}$ at $37^{\circ} \mathrm{C}$. Samples were taken after 0 , $1,2,4,6$, and $24 \mathrm{~h}$. As negative control either potassium phosphate buffer, AF without lysozyme, or 1:10 diluted $\mathrm{WF}_{\mathrm{A}}$ were used corresponding to the tested sample. For each time point samples were 


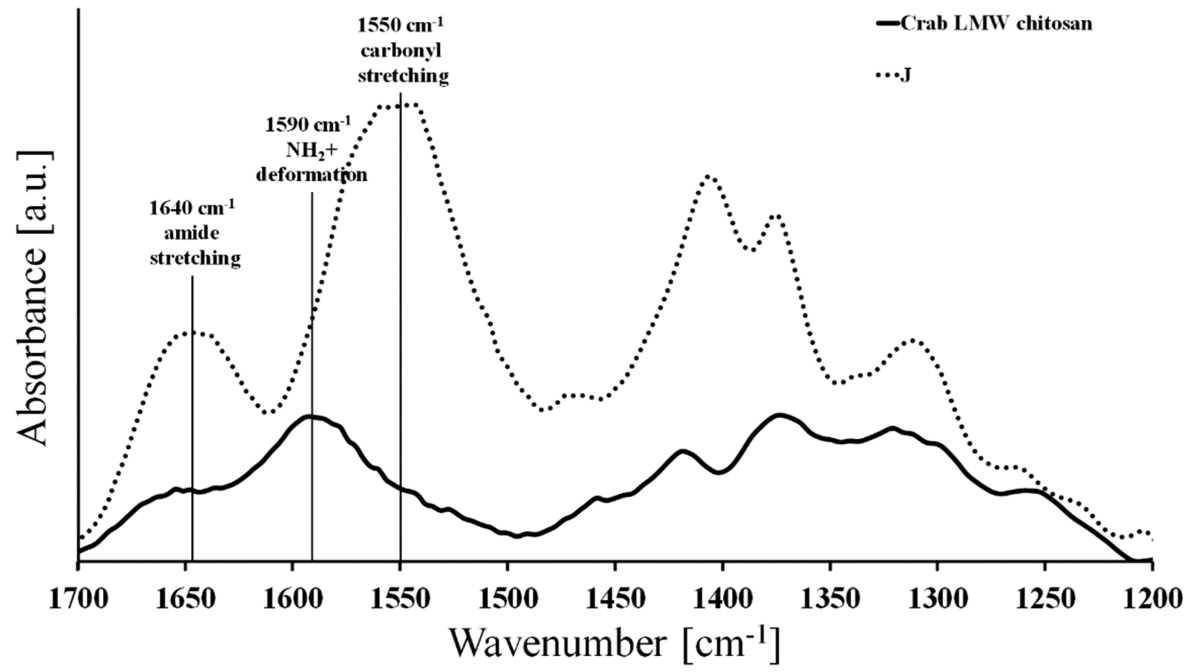

Figure 1. ATR-FTIR spectra (normalized in the region $1025 \mathrm{~cm}^{-1}$ and baseline-corrected) of native crab LMW chitosans, and $N$-acetylated crab LMW chitosans (sample J), whereby the increase in the absorbance of the amide stretching band is at $1640 \mathrm{~cm}^{-1}$ and the carbonyl stretching band at $1540 \mathrm{~cm}^{-1}$.
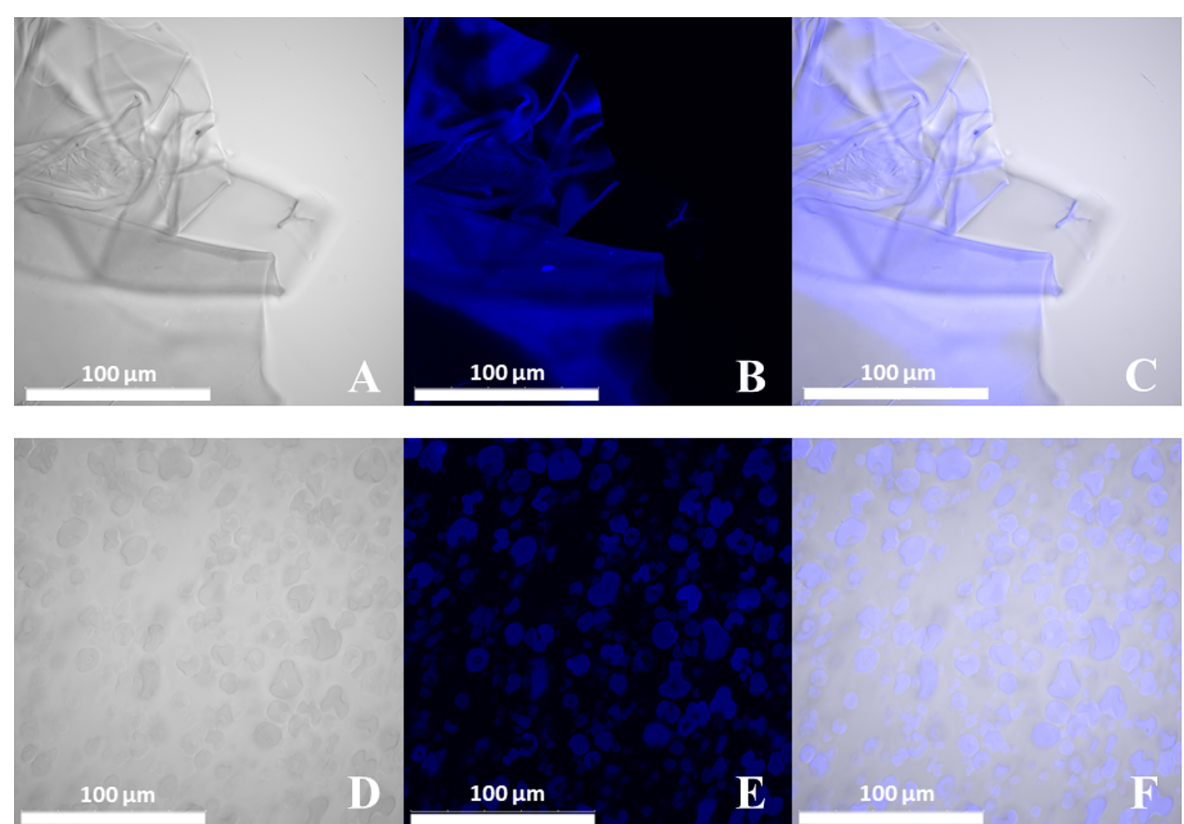

Figure 2. Confocal laser scanning microscope images of RB5-stained $N$-acetylated crab LMW chitosan flakes of sample $\mathbf{J}$ of (A) the bright-field channel, (B) the fluorescent channel (laser light wavelength of $405 \mathrm{~nm}, \lambda_{\mathrm{em}} 426-526 \mathrm{~nm}$ ), and (C) the corresponding overlay. Images of the RB5stained spray-dried $\mathrm{N}$-acetylated chitosan particles $\mathrm{J}_{\mathrm{SD}}$ of (D) the bright-field channel, (E) the fluorescent channel (laser light wavelength of 405 $\mathrm{nm}, \lambda_{\mathrm{em}} 426-526 \mathrm{~nm}$ ), and (F) the corresponding overlay. Scale bar refers to $100 \mu \mathrm{m}$.

centrifuged, and $200 \mu \mathrm{L}$ of the supernatant was removed for $\mathrm{UV} /$ vis measurements at $597 \mathrm{~nm}$ (absorbance maxima of RB5) using a Tecan Infinite 200 Pro plate reader (Tecan Trading AG). After the measurements, the aliquots were returned to the reaction mix for further incubation.

\section{RESULTS AND DISCUSSION}

Lysozyme has been previously investigated as a potential biomarker for the detection of wound infections. ${ }^{15,19}$ Strategies involving peptidoglycan from bacterial cell walls as lysozyme substrate showed limitations due to the risk of direct contact to patient wounds potentially inducing immune responses. Circumventing this bottleneck, detection systems based on chitosans were previously published including composite material wound dressings, ${ }^{28-30}$ hydrogels, ${ }^{31}$ and particles. ${ }^{32,19}$
However, there is a need for materials allowing a simple and flexible integration into a variety of sensor systems. Hence, the formulation of spray-dried $\mathrm{N}$-acetylated chitosan particles was hypothesized to further improve lysozyme-induced hydrolysis of chitosan for the application in wound infection detection. Hereby, spray-drying and the corresponding increase of the surface area to volume ratio due to the formation of atomized droplets and water evaporation is described as beneficial for the increase in release properties of the material. ${ }^{33-35}$ Moreover, the higher surface area to volume ratio can facilitate the contact and penetration with buffer, as previously reported by Panyam et al., ${ }^{36}$ or within this study with wound fluid.

Characterization of Raw Materials and $\mathbf{N}$-Acetylation

Products. Native chitosans derived from crab (MMW and 
LMW) and shrimp shells were investigated regarding their molecular weights using SEC-MALLS. High deviations based on the origin of the used chitosan were observed, ranging from $485.5 \pm 3.7 \mathrm{kDa}$ (crab MMW) over $236.69 \pm 4.5 \mathrm{kDa}$ (shrimp) to $121.8 \pm 7.0 \mathrm{kDa}$ (crab LMW) for the numberaverage molecular weights $\left(M_{n}\right)$ (see the SI, Figure S1A) (Table 1); the weight-average molecular weights $\left(M_{\mathrm{w}}\right)$ showed comparable results (SI, Figure S1B). Similar polydispersity values (SI, Figure S1C) were obtained for chitosans of all tested origins.

The $N$-acetylated chitosans were analyzed by ${ }^{1} \mathrm{H}$ NMR (Table 1, Figure S2) and ATR-FTIR (Figure 1) showing significant shifts in the amide carbonyl stretching band (1640 $\left.\mathrm{cm}^{-1}\right)$, the $\mathrm{NH}_{2}^{+}$deformation band $\left(1590 \mathrm{~cm}^{-1}\right)$, and the amide $\mathrm{N}-\mathrm{H}$ stretching band $\left(1540 \mathrm{~cm}^{-1}\right)$ indicating successful $N$-acetylation.

${ }^{1} \mathrm{H}$ NMR spectra showed that $N$-acetylation with 1.0 (shrimp) and 1.25 (crab LMW, shrimp and crab MMW) mol equiv of acetic anhydride was successful (Table 1); however, the calculation of the DA was not possible because of insoluble fractions of synthesis products during sample preparation for ${ }^{1} \mathrm{H}$ NMR. $N$-Acetylation using $0.50,0.75$, and 1.00 mol equiv resulted in DAs between $36 \%$ and $51 \%$, whereby the increase in applied acetic anhydride showed the expected corresponding increase in DA values within one type of chitosan. An impact of the chitosan's origin on the DA could not be determined when comparing the DA results for shrimp, crab MMW, or crab LMW using equivalent amounts of acetic anhydride. On the basis of these findings, all successfully acetylated chitosans were used for further functionalization, i.e., staining with RB5, for the optimization regarding early wound infection detection and therefore for spray-drying and lysozyme response.

$\mathrm{N}$-Acetyl Chitosan Functionalization and Solubility Studies. $N$-Acetylation of chitosans was previously described to be a suitable method for the increase of the lysozyme hydrolysis rate of this naturally derived biopolymer. ${ }^{37} \mathrm{~N}$ Acetylated chitosans were labeled with RB5 to allow a simple visual detection of lysozyme activity based on the release of stained fragments from the functionalized polymer. After staining, $\mathrm{N}$-acetylated chitosan substrates were tested for their solubility and suitability for spray-drying (Table 1). For the labeling process, reactive black 5 was chosen, a reactive vinylsulfone dye forming a vinyl intermediate under basic conditions, which subsequently reacts with a given nucleophile. Using this approach, previous labeling studies using RB5 revealed the best results regarding leaching and homogeneity. ${ }^{19}$ Therefore, RB5 labeling was conducted, and the previous published results could be confirmed within this study using CLSM to investigate the homogeneity of dye saturation of the $\mathrm{N}$-acetyl chitosan substrates (Figure $2 \mathrm{~A}-\mathrm{C}$ ). Images showed flake-shaped structures of sample $\mathrm{J}$ before spray-drying, whereby fluorescent images confirmed the homogeneous dye distribution over the complete material. Despite the fact that homogeneous staining was observed for all $\mathrm{N}$-acetylated chitosan samples, only sample $\mathbf{J}$ showed complete solubility in $1 \mathrm{wt} \%$ acetic acid solution in water required for spraydrying.

Enzyme Activity of Lysozyme and Wound Fluids. The enzyme activity for lysozyme of hen egg white with 846.67 $\mu \mathrm{kat} / \mathrm{mg}$, noninfected $\left(\mathrm{WF}_{\mathrm{A}}\right)$ and infected $\left(\mathrm{WF}_{\mathrm{B}}\right)$ wound fluids was tested using an enzyme activity assay based on the method of Shugar et al. ${ }^{27}$ The obtained activities were considerably different for lysozyme, noninfected $33.89 \pm$ $5.36 \mu \mathrm{kat} / \mathrm{mL} \mathrm{WF}_{\mathrm{A}}$, and infected $\mathrm{WF}_{\mathrm{B}} 301.17 \pm 5.53 \mu \mathrm{kat} / \mathrm{mL}$ confirming the potential for infection detection.

Spray-Drying and Characterization of Product. Spraydrying to microparticles is described as a complex process fully controlling the mechanism of component radial distribution during the drying process. Additionally, surface activity is seen as a major driving factor for successful spray-drying, as it can lead to preferential absorption of components on the droplet surface which can cause a diffusional flux toward the surface. ${ }^{38}$ Accordingly, spray-drying of solubilized sample $\mathbf{J}$ was performed, and the obtained spray-dried particles $\left(\mathrm{J}_{\mathrm{SD}}\right)$ were characterized regarding particle size, surface potential and aggregation behavior, morphology, and staining homogeneity.

The particle size was analyzed in potassium phosphate buffer $\mathrm{pH} 6.2$, mimicking the conditions of AWF and WF to a minor degree, and showed an average hydrodynamic radius of 612.5 $\pm 132.8 \mathrm{~nm}$, indicating that a submicron fraction is present (Figure 3). However, RB5-stained spray-dried particles ( $\mathbf{J}_{\mathrm{SD}}$ )

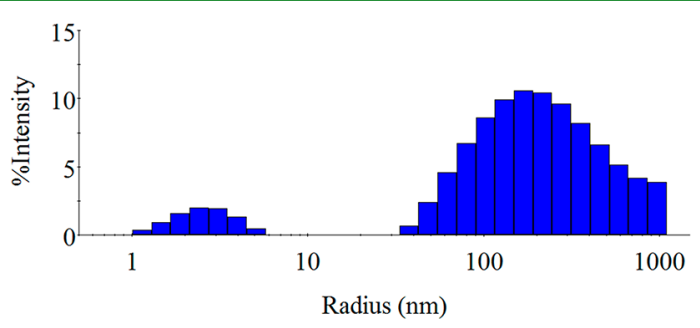

Figure 3. Intensity distribution obtained via dynamic light scattering of spray-dried $N$-acetylated crab LMW chitosans $\left(\mathrm{J}_{\mathrm{SD}}\right)$ measured in 66 $\mathrm{mM}$ potassium phosphate buffer $\mathrm{pH} 6.2$ showing two significant different particle species: species $1 R_{\mathrm{H}}$ about $4 \mathrm{~nm}$; species $2 R_{\mathrm{H}}$ about $200 \mathrm{~nm}$.

showed a large microfraction (Figure 4); DLS measurements are limited to a submicron range. Therefore, size information was additionally obtained by SEM and CLSM analysis. Additionally, the intense blue color of $\mathbf{J}_{S D}$ particles acts as a limiting factor for DLS experiments resulting in high standard deviations.

The intensity distribution of $\mathbf{J}_{\mathbf{S D}}$ revealed a polydisperse behavior, whereby a larger particle species dominates the distribution, as two significant different particle species can be observed (species $1 R_{\mathrm{H}}$ about $4 \mathrm{~nm}$; species $2 R_{\mathrm{H}}$ about 200 $\mathrm{nm}$ ) in Figure 3. However, SEM (Figure 4) and CLSM images (Figure 2) showed no agglomeration or aggregation of the particles.

SEM images showed sample heterogeneity with particle sizes ranging between $244.6 \mathrm{~nm}$ and $4.38 \mu \mathrm{m}$, which supports the DLS data regarding the presence of a submicron fraction, as well as structural information. Microscopy-based particle size was evaluated separately for the submicron and the microfraction (Figure 5) of $\mathbf{J}_{\mathbf{S D}}$ particles. The calculated average particle radius was $617.8 \pm 194.0 \mathrm{~nm}$; however, particles with sizes smaller than $200 \mathrm{~nm}$ could not be observed because of occurring image resolution limits and corresponding blur at higher magnifications caused by instrument limitations of the SEM instrument used. Further higher magnifications and therefore higher energies negatively affected sample stability leading to significant particle destruction; as a result images were obtained at magnifications of $100 \times, 1000 \times$, and $2000 \times$. In direct comparison obtained SEM- and DLS-based radii showed significant comparability, further supported by the 

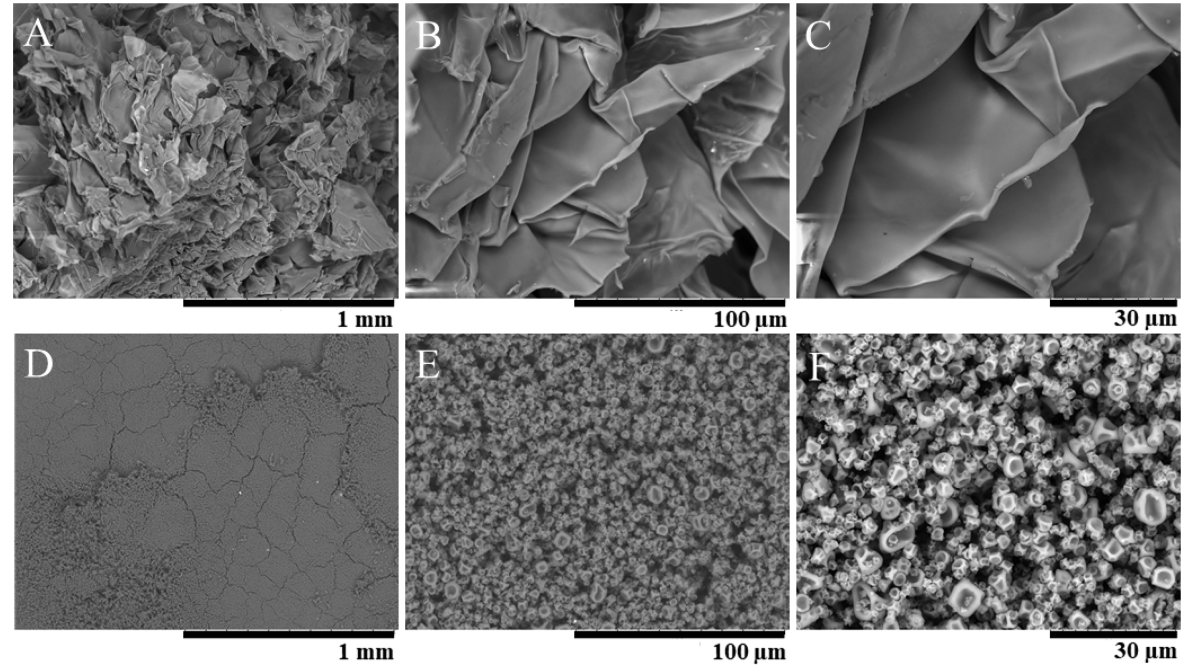

Figure 4. Scanning electron microscopic images of RB5-stained $N$-acetylated crab LMW chitosan flakes of sample J with (A) 100×, (B) 1000×, and (C) $2000 \times$ magnification and images of the RB5-stained $N$-acetylated crab LMW chitosan spray-dried particles of sample $\mathbf{J}_{S D}$ with (D) $100 \times$, (E) $1000 \times$, and (F) 2000× magnification.

A

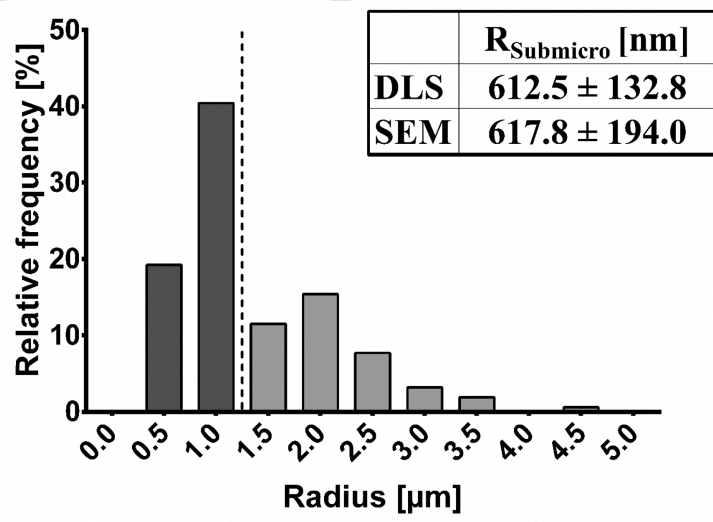

Figure 5. (A) Calculated relative frequency distribution based on scanning electron microscopic images of RB5-stained spray-dried $\mathrm{N}$ acetylated chitosan particles (sample $\mathbf{J}_{\mathbf{S D}}$ ) processed with Image software including the submicron and microfraction of the sample (delimited with dotted line). (B) Obtained radii of RB5-stained spraydried $N$-acetylated chitosan particles $\left(\mathrm{J}_{\mathrm{SD}}\right)$ using DLS (hydrodynamic radius in $\mathrm{nm}$ ) and scanning electron microscopy (radius in $\mathrm{nm}$ ) of the submicron fraction.

displayed intensity distribution (Figure 3) and the relative frequency distribution (Figure 5). In general, the presence of the submicron fraction is seen as highly benefiting because of the increased surface area when compared to the same volume of the materials. This effect was previously described by Biener et $\mathrm{al}^{39}$ and leads to a hypothesized enhanced reaction possibility due to the elevated potential for lysozyme to interact with the presented $\mathrm{N}$-acetylated chitosan particle surface compared to the significant smaller surface of microparticles of the same volume. Therefore, the application of $\mathbf{J}_{\mathrm{SD}}$ particles in future in vitro test systems should offer rapid infection detection due to the enhanced reaction possibility and hence reaction velocity.

$\mathrm{J}_{\mathrm{SD}}$ particles were spherically shaped with porous fracture surfaces showing significant topologies. Porous fracture surfaces of the particles are hypothesized to lead to an enhanced lysozyme-responsive release of RB5 due to the increased contact area for lysozyme attack based on the increase of the surface area to volume ratio compared to $N$ acetylated chitosan flakes before spray-drying. ${ }^{36,38}$ Therefore, stain distribution was further investigated after spray-drying by using CLSM (Figure 2). Compared to the homogeneous staining before spray-drying, $\mathbf{J}_{\text {SD }}$ particles showed a highly homogeneous labeling, whereby surface topology impacts where visible. Additionally, $\zeta$ potential measurements were performed analyzing the aggregation behavior and particle stability (Figure 6).

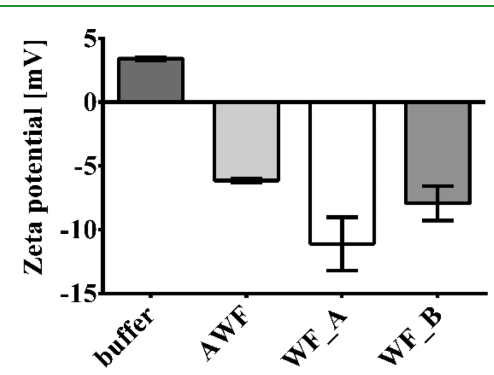

Figure 6. $\zeta$ potential of spray-dried $N$-acetylated crab LMW chitosan particle sample $\mathbf{J}_{\mathrm{SD}}$ measured in $66 \mathrm{mM}$ potassium phosphate buffer $\mathrm{pH}$ 6.2, artificial wound fluid with a lysozyme activity of $83.33 \mu \mathrm{kat} /$ $\mathrm{mg}$, and wound fluid with low $\left(\mathrm{WF}_{\mathrm{A}}, 33.89 \pm 5.36 \mu \mathrm{kat} / \mathrm{mL}\right)$ and high $\left(\mathrm{WF}_{\mathrm{B}}, 301.17 \pm 5.53 \mu \mathrm{kat} / \mathrm{mL}\right)$ lysozyme activity.

A $\zeta$ potential $(\zeta)$ between $+3.42 \pm 0.12$ and $-11.11 \pm 2.09$ $\mathrm{mV}$ was obtained, highly influenced by the used solvent (buffer, $\mathrm{AWF}, \mathrm{WF}_{\mathrm{A}}$, or $\mathrm{WF}_{\mathrm{B}}$ ). $\zeta$ values between -10 and -20 $\mathrm{mV}$ are considered to refer to relatively stable particles. ${ }^{40}$ However, as previously discussed, no aggregation behavior could be seen in SEM and CLSM studies (Figures 2 and 4). Ionic-strength-induced changes from positive to negative $\zeta$ are due to the compression of the electric double layer induced by an increasing presence of ions. ${ }^{40}$ This effect can be clearly seen comparing the $\zeta$ in potassium phosphate buffer $\mathrm{pH} 6.2(+3.42$ $\pm 0.12 \mathrm{mV})$ and AWF $(-6.14 \pm 0.16 \mathrm{mV})$ or WF $\left(\mathrm{WF}_{\mathrm{A}}\right.$ $\left.-11.11 \pm 2.09 \mathrm{mV} ; \mathrm{WF}_{\mathrm{B}}-7.93 \pm 1.35 \mathrm{mV}\right)$. AWF has higher ionic strength due to the composition compared to the potassium phosphate buffer; however, compared to the values 
A

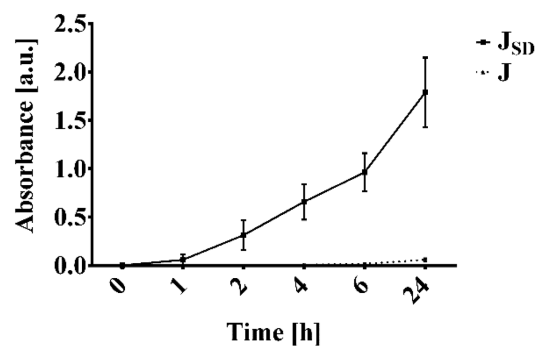

B

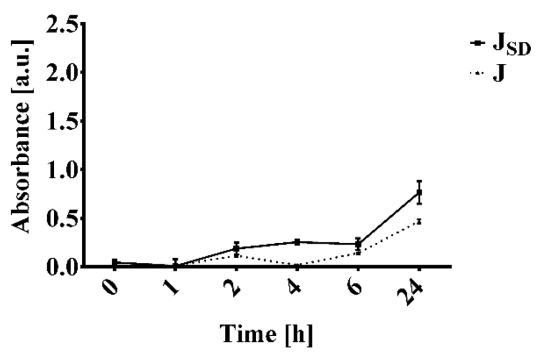

C

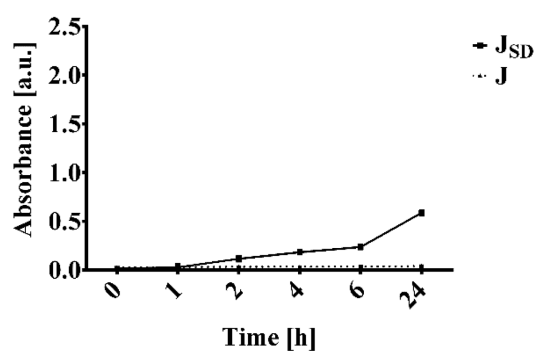

Figure 7. Lysozyme-responsive release of RB5 from stained $N$-acetylated crab LMW chitosan sample $\mathbf{J}$ before and after spray-drying ( $\mathrm{J}_{\text {SD }}$ ). (A) Increase of absorbance [a.u.] at $597 \mathrm{~nm}$ over an incubation time of $24 \mathrm{~h}$ in $66 \mathrm{mM}$ potassium phosphate buffer pH 6.2 with a concentration of 1 $\mathrm{mg} / \mathrm{mL}$ lysozyme $(83.33 \mu \mathrm{kat} / \mathrm{mg})$. (B) Increase of absorbance [a.u.] at $597 \mathrm{~nm}$ over an incubation time of $24 \mathrm{~h}$ in artificial wound fluid with a concentration of $1 \mathrm{mg} / \mathrm{mL}$ lysozyme $(83.33 \mu \mathrm{kat} / \mathrm{mg})$. (C) Release profile displayed as increase of absorbance [a.u.] over time [h] at $597 \mathrm{~nm}$ in positive wound fluid $\left(\mathrm{WF}_{\mathrm{B}}\right)$ with an activity of $301.17 \pm 5.53 \mu \mathrm{kat} / \mathrm{mL}$.

measured in $\mathrm{WF}$, the ionic strength of infected $\mathrm{WF}_{\mathrm{B}}$ is lower than that of noninfected $\mathrm{WF}_{\mathrm{A}}$. This effect can be caused by the high activity of lysozyme and other enzymes (MPO, HNE) present in infected WF and the enzyme surface charges, ${ }^{41}$ which show a significant impact on the ionic strength. ${ }^{42}$ This theory is supported by the comparison of the AWF (lysozyme activity of $83.33 \mu \mathrm{kat} / \mathrm{mg}$ ) with noninfected $\mathrm{WF}_{\mathrm{A}}$ (lysozyme activity of $33.89 \pm 5.36 \mu \mathrm{kat} / \mathrm{mL}$ ), whereby the higher lysozyme activity in AWF also possibly leads to a decrease in the $\zeta$ compared to $\mathrm{WF}_{\mathrm{A}}$. Furthermore, for later applications as an infection detection system, negative $\zeta$ values are seen as beneficial regarding the reduction of possible arterial clogging induced by direct wound contact in case of mishandling, which was recently reported by Honary and Zahir ${ }^{43}$ and Sonavane et al. $^{44}$

Lysozyme Detection. On the basis of the aimed approach applying $\mathbf{J}_{S D}$ particles for early-stage wound infection detection, the lysozyme-mediated release of RB5-stained fragments was investigated in potassium phosphate buffer $\mathrm{pH}$ 6.2, AWF, and infected $\mathrm{WF}_{\mathrm{B}}$ (Figure 7). Despite slightly faster hydrolysis at $\mathrm{pH} 5$ compared to $\mathrm{pH} 6.2$ which was previously reported using $\mathrm{N}$-acetylated shrimp chitosans, ${ }^{19}$ HMC-based $\mathbf{J}_{S D}$ particles showed complete dissolution in $\mathrm{pH} 6.2$ after $24 \mathrm{~h}$ of incubation.

In general, spray-drying increased the release behavior for all tested solvents, whereby maximum RB5 release was observed after incubation of $\mathbf{J}_{\mathrm{SD}}$ particles in $66 \mathrm{mM}$ potassium phosphate buffer $\mathrm{pH} 6.2$ with a lysozyme activity of $83.33 \mu \mathrm{kat} / \mathrm{mg}$. Enhanced lysozyme-mediated release of RB5 from $\mathbf{J}_{S D}$ particles might result from the increased surface area. The increase of the surface/volume ratio can be associated with the facilitation of buffer contact and penetration into the particles. Furthermore, faster diffusion of monomers and oligomers out of the particles is simultaneously enhanced, which can be compared to results previously reported by Panyam et al. for spray-dried poly(lactic-co-glycolic) acid particles. ${ }^{36}$ On the other hand, the random distribution of the $\mathrm{N}$-acetyl groups could provide a substantial amount of lysozyme-binding sites. ${ }^{45}$ Lysozyme-responsive release in $\mathrm{AWF}$ and $\mathrm{WF}_{\mathrm{B}}$ showed comparable results, whereas the release of stained chitosan was decreased incubating in lysozyme containing potassium phosphate buffer $\mathrm{pH}$ 6.2. These data correlate to the results of laser Doppler velocimetry, since the $\zeta$ showed a significant impact on lysozyme-binding. The reduced ionic strength due to the possible attachment of ions on the enzyme surface, e.g., in the case of AWF chloride or lactate, is hypothesized to reduce the binding properties, which leads to a decrease in released RB5 from $\mathbf{J}_{S D}$ particles. Nonetheless, the release studies tested in WF of infected wounds led to positive results regarding the enhancement of fast lysozyme-mediated release using the spray-drying particles, showing a 5-fold increase compared to our previous studies. ${ }^{19}$ Therefore, the application in a rapid in vitro standalone infection detection approach is seen as promising, offering fast detection without direct wound contact of the material.

\section{CONCLUSIONS}

In this study, chitosans of different physicochemical properties were chemically functionalized and tested upon their suitability for particle formation by spray-drying. Reactive black-5-stained crab LMW $N$-acetyl chitosan substrates were successfully spray-dried, and the obtained particles showed an average hydrodynamic radius of $612.5 \pm 132.8 \mathrm{~nm}$, indicating that a submicron fraction is present. The $\zeta$ potential and surface charge were investigated in relation to later applications as an infection detection system for wound infections, whereby the negative ZP indicated a high biocompatibility. Furthermore, aggregation behavior and surface structure were analyzed revealing spherical-shaped particles with explicit surface topologies. The obtained increased surface area to volume 
ratio correlated to the results of the in vitro release studies. Hereby, spray-dried $N$-acetyl chitosan particles showed a 5 times faster lysozyme-mediated release of reactive black 5. On the basis of the results, this approach showed the potential for the application of spray-dried $N$-acetyl chitosan particles for wound-fluid-based in vitro early infection detection. The spraydried lysozyme substrate greatly improved the stability of the detection material, thus facilitating its integration in different infection detection systems such as rapid in vitro standalone devices without direct patient contact.

\section{ASSOCIATED CONTENT}

\section{S Supporting Information}

The Supporting Information is available free of charge on the ACS Publications website at DOI: 10.1021/acsabm.9b00023.

SEC-MALLS analysis of native chitosans, and ${ }^{1} \mathrm{H}$ NMR analysis of $N$-acetylated crab LMW chitosans (PDF)

\section{AUTHOR INFORMATION}

\section{Corresponding Author}

*E-mail: gregor.tegl@gmail.com.

\section{ORCID $\odot$}

Gregor Tegl: 0000-0002-4242-1693

Notes

The authors declare no competing financial interest.

\section{ACKNOWLEDGMENTS}

Wound fluids and facilities for wound fluid studies were kindly provided by Qualizyme Diagnostics $\mathrm{GmbH}$. We also want to thank Karin Wieland (Vienna University of Technology, Vienna, Austria) for scientific input on FTIR result interpretation and Clemens Gamerith (ACIB GmbH, Graz, Austria) for technical support during wound fluid studies. This work was performed within the European project Infact and has received financial funding from the European FP7programme Grant Agreement 604278 and from the NÖ Forschungs- und Bildungsges.m.b.H. (NFB) and the provincial government of Lower Austria through the Science Calls (Project ID: SC16-024). The Austrian Centre of Industrial Biotechnology (ACIB) is gratefully acknowledged. A.P. thanks the FWF Erwin Schrödinger fellowship (Grant Agreement: J 4014-N34) for financial support.

\section{REFERENCES}

(1) MedMarket Diligence. Worldwide Wound Management, Forecast to 2026: Established and Emerging Products, Technologies and Markets in the Americas, Europe, Asia/Pacific and Rest of World; 2018.

(2) Sen, C. K.; Gordillo, G. M.; Roy, S.; Kirsner, R.; Lambert, L.; Hunt, T. K.; Gottrup, F.; Gurtner, G. C.; Longaker, M. T. Human Skin Wounds: A Major and Snowballing Threat to Public Health and the Economy. Wound Repair Regen. 2009, 17 (6), 763-771.

(3) F. Gottrup, A.; Melling, D. A. H. An Overview of Surgical Site Infections: Aetiology, Incidence and Risk Factors. http://www. worldwidewounds.com/2005/september/Gottrup/Surgical-SiteInfections-Overview.html (accessed May 26, 2015).

(4) Kawasumi, A.; Sagawa, N.; Hayashi, S.; Yokoyama, H.; Tamura, $\mathrm{K}$. Wound Healing in Mammals and Amphibians: Toward Limb Regeneration in Mammals. Curr. Top. Microbiol. Immunol. 2012, 367, 33-49.

(5) Bowler, P. G.; Duerden, B. I.; Armstrong, D. G. Wound Microbiology and Associated Approaches to Wound Management. Clin. Microbiol. Rev. 2001, 14 (2), 244-269.
(6) Schiffer, D.; Verient, V.; Luschnig, D.; Blokhuis-Arkes, M. H. E.; Palen, J. V. D.; Gamerith, C.; Burnet, M.; Sigl, E.; Heinzle, A.; Guebitz, G. M. Lysozyme-Responsive Polymer Systems for Detection of Infection. Eng. Life Sci. 2015, 15 (4), 368-375.

(7) Williams, D. T.; Hilton, J. R.; Harding, K. G. Diagnosing Foot Infection in Diabetes. Clin. Infect. Dis. 2004, 39 (S2), S83-S86.

(8) Cutting, K. F.; Harding, K. G. Criteria for Identifying Wound Infection. J. Wound Care 1994, 3 (4), 198-201.

(9) Gardner, S. E.; Frantz, R. a; Doebbeling, B. N. The Validity of the Clinical Signs and Symptoms Used to Identify Localized Chronic Wound Infection. Wound Repair Regen. 2001, 9 (3), 178-186.

(10) Gardner, S. E.; Frantz, R. A.; Saltzman, C. L.; Hillis, S. L.; Park, H.; Scherubel, M. Diagnostic Validity of Three Swab Techniques for Identifying Chronic Wound Infection. Wound Repair Regen. 2006, 14 (5), 548-557.

(11) Yager, D. R.; Kulina, R. A.; Gilman, L. A. Wound Fluids: A Window into the Wound Environment? Int. J. Lower Extremity Wounds 2007, 6 (4), 262-272.

(12) Tegl, G.; Schiffer, D.; Sigl, E.; Heinzle, A.; Guebitz, G. M. Biomarkers for Infection: Enzymes, Microbes, and Metabolites. Appl. Microbiol. Biotechnol. 2015, 99 (11), 4595-4614.

(13) World Union of Wound Healing Societies (WUWHS). Principles of Best Practice: Diagnostics and Wounds. A Consenus Document; MEP Ltd.: London, 2008.

(14) Torsteinsdóttir, I.; Hâkansson, L.; Hällgren, R.; Gudbjörnsson, B.; Arvidson, N. G.; Venge, P. Serum Lysozyme: A Potential Marker of Monocyte/Macrophage Activity in Rheumatoid Arthritis. Rheumatology (Oxford, U. K.) 1999, 38 (12), 1249-1254.

(15) Hasmann, A.; Wehrschuetz-Sigl, E.; Kanzler, G.; Gewessler, U.; Hulla, E.; Schneider, K. P.; Binder, B.; Schintler, M.; Guebitz, G. M. Novel Peptidoglycan-Based Diagnostic Devices for Detection of Wound Infection. Diagn. Microbiol. Infect. Dis. 2011, 71 (1), 12-23.

(16) Hasmann, A.; Gewessler, U.; Hulla, E.; Schneider, K. P.; Binder, B.; Francesko, A.; Tzanov, T.; Schintler, M.; Van der Palen, J.; Guebitz, G. M.; Wehrschuetz-Sigl, E. Sensor Materials for the Detection of Human Neutrophil Elastase and Cathepsin G Activity in Wound Fluid. Exp. Dermatol. 2011, 20 (6), 508-513.

(17) Stokke, B. T.; Vårum, K. M.; Holme, H. K.; Hjerde, R. J. N.; Smidsrød, O. Sequence Specificities for Lysozyme Depolymerization of Partially N -Acetylated Chitosans. Can. J. Chem. 1995, 73 (11), $1972-1981$

(18) Han, T.; Nwe, N.; Furuike, T.; Tokura, S.; Tamura, H. Methods of N -Acetylated Chitosan Scaffolds and Its in Vitro Biodegradation by Lysozyme. J. Biomed. Sci. Eng. 2012, 5, 15-23.

(19) Tegl, G.; Rollett, A.; Dopplinger, J.; Gamerith, C.; Guebitz, G. M. Chitosan Based Substrates for Wound Infection Detection Based on Increased Lysozyme Activity. Carbohydr. Polym. 2016, 151, 260267.

(20) Riva, R.; Ragelle, H.; des Rieux, A.; Duhem, N.; Jérôme, C.; Préat, V. Chitosan and Chitosan Derivatives in Drug Delivery and Tissue Engineering; Springer: Berlin Heidelberg, 2011; pp 19-44.

(21) Kean, T.; Thanou, M. Biodegradation, Biodistribution and Toxicity of Chitosan. Adv. Drug Delivery Rev. 2010, 62 (1), 3-11.

(22) Amidi, M.; Mastrobattista, E.; Jiskoot, W.; Hennink, W. E. Chitosan-Based Delivery Systems for Protein Therapeutics and Antigens. Adv. Drug Delivery Rev. 2010, 62 (1), 59-82.

(23) Agnihotri, S. A.; Mallikarjuna, N. N.; Aminabhavi, T. M. Recent Advances on Chitosan-Based Micro- and Nanoparticles in Drug Delivery. J. Controlled Release 2004, 100 (1), 5-28.

(24) Schneider, C. A.; Rasband, W. S.; Eliceiri, K. W. NIH Image to ImageJ: 25 Years of Image Analysis. Nat. Methods 2012, 9 (7), 671675.

(25) Nogueira, E.; Gomes, A. C.; Preto, A.; Cavaco-Paulo, A. Design of Liposomal Formulations for Cell Targeting. Colloids Surf., B 2015, $136,514-526$.

(26) Sze, A.; Erickson, D.; Ren, L.; Li, D. Zeta-Potential Measurement Using the Smoluchowski Equation and the Slope of the Current-time Relationship in Electroosmotic Flow. J. Colloid Interface Sci. 2003, 261 (2), 402-410. 
(27) Shugar, D. The Measurement of Lysozyme Activity and the Ultra-Violet Inactivation of Lysozyme. Biochim. Biophys. Acta 1952, 8 (3), 302-309.

(28) Aoyagi, S.; Onishi, H.; Machida, Y. Novel Chitosan Wound Dressing Loaded with Minocycline for the Treatment of Severe Burn Wounds. Int. J. Pharm. 2007, 330 (1-2), 138-145.

(29) Wang, T.; Zhu, X.-K.; Xue, X.-T.; Wu, D.-Y. Hydrogel Sheets of Chitosan, Honey and Gelatin as Burn Wound Dressings. Carbohydr. Polym. 2012, 88 (1), 75-83.

(30) Mi, F.-L.; Wu, Y.-B.; Shyu, S.-S.; Schoung, J.-Y.; Huang, Y.-B.; Tsai, Y.-H.; Hao, J.-Y. Control of Wound Infections Using a Bilayer Chitosan Wound Dressing with Sustainable Antibiotic Delivery. J. Biomed. Mater. Res. 2002, 59 (3), 438-449.

(31) Sudheesh Kumar, P. T.; Lakshmanan, V.-K.; Anilkumar, T. V.; Ramya, C.; Reshmi, P.; Unnikrishnan, A. G.; Nair, S. V.; Jayakumar, R. Flexible and Microporous Chitosan Hydrogel/Nano $\mathrm{ZnO}$ Composite Bandages for Wound Dressing: In Vitro and In Vivo Evaluation. ACS Appl. Mater. Interfaces 2012, 4 (5), 2618-2629.

(32) Ye, W.; Leung, M. F.; Xin, J.; Kwong, T. L.; Lee, D. K. L.; Li, P. Novel Core-Shell Particles with Poly(n-Butyl Acrylate) Cores and Chitosan Shells as an Antibacterial Coating for Textiles. Polymer 2005, 46 (23), 10538-10543.

(33) Gharsallaoui, A.; Roudaut, G.; Chambin, O.; Voilley, A.; Saurel, R. Applications of Spray-Drying in Microencapsulation of Food Ingredients: An Overview. Food Res. Int. 2007, 40 (9), 1107-1121.

(34) Yang, Y.-Y.; Chung, T.-S.; Ping Ng, N. Morphology, Drug Distribution, and in Vitro Release Profiles of Biodegradable Polymeric Microspheres Containing Protein Fabricated by Double-Emulsion Solvent Extraction/Evaporation Method. Biomaterials 2001, 22 (3), 231-241.

(35) Elversson, J.; Millqvist-Fureby, A. Particle Size and Density in Spray Drying-Effects of Carbohydrate Properties. J. Pharm. Sci. 2005, 94 (9), 2049-2060.

(36) Panyam, J.; Dali, M. M.; Sahoo, S. K.; Ma, W.; Chakravarthi, S. S.; Amidon, G. L.; Levy, R. J.; Labhasetwar, V. Polymer Degradation and in Vitro Release of a Model Protein from Poly(d,l-Lactide-CoGlycolide) Nano- and Microparticles. J. Controlled Release 2003, 92 (1-2), 173-187.

(37) Zhang, Y.; Wang, Z.; Zhang, J.; Chen, C.; Wu, Q.; Zhang, L.; Zhang, X. Quantitative Determination of Chitinolytic Activity of Lysozyme Using Half-Deacetylated Chitosan as a Substrate. Carbohydr. Polym. 2011, 85 (3), 554-559.

(38) Vehring, R. Pharmaceutical Particle Engineering via Spray Drying. Pharm. Res. 2008, 25 (5), 999-1022.

(39) Biener, J.; Wittstock, A.; Baumann, T.; Weissmüller, J.; Bäumer, M.; Hamza, A. Surface Chemistry in Nanoscale Materials. Materials 2009, 2 (4), 2404-2428.

(40) Bhattacharjee, S. DLS and Zeta Potential - What They Are and What They Are Not? J. Controlled Release 2016, 235, 337-351.

(41) Kuehner, D. E.; Engmann, J.; Fergg, F.; Wernick, M.; Blanch, H. W.; Prausnitz, J. M. Lysozyme Net Charge and Ion Binding in Concentrated Aqueous Electrolyte Solutions. J. Phys. Chem. B 1999, 103, 1368-1374.

(42) Steudle, A.; Pleiss, J. Modelling of Lysozyme Binding to a Cation Exchange Surface at Atomic Detail: The Role of Flexibility. Biophys. J. 2011, 100 (12), 3016-3024.

(43) Honary, S.; Zahir, F. Effect of Zeta Potential on the Properties of Nano-Drug Delivery Systems - A Review (Part 2). Trop. J. Pharm. Res. 2013, 12 (2), 265-273.

(44) Sonavane, G.; Tomoda, K.; Makino, K. Biodistribution of Colloidal Gold Nanoparticles after Intravenous Administration: Effect of Particle Size. Colloids Surf., B 2008, 66 (2), 274-280.

(45) Stokke, B. T.; Vårum, K. M.; Holme, H. K.; Hjerde, R. J. N.; Smidsrød, O. Sequence Specificities for Lysozyme Depolymerization of Partially N -Acetylated Chitosans. Can. J. Chem. 1995, 73 (11), 1972-1981. 